\title{
On the non-extendibility of strongness and supercompactness through strong compactness
}

by

\author{
Arthur W. Apter (New York, NY)
}

\begin{abstract}
If $\kappa$ is either supercompact or strong and $\delta<\kappa$ is $\alpha$ strong or $\alpha$ supercompact for every $\alpha<\kappa$, then it is known $\delta$ must be (fully) strong or supercompact. We show this is not necessarily the case if $\kappa$ is strongly compact.
\end{abstract}

1. Introduction and preliminaries. A well-known result of Magidor [16] states that if $\kappa$ is supercompact and $\delta<\kappa$ is $\alpha$ supercompact for all $\alpha<\kappa$, then $\delta$ is supercompact. Indeed, the following is true.

LEMma 1.1 (Folklore). If $\kappa$ is a strong cardinal and $\delta<\kappa$ is either $\alpha$ strong, $\alpha$ strongly compact, or $\alpha$ supercompact for every $\alpha<\kappa$, then $\delta$ must be (fully) strong, strongly compact, or supercompact.

Proof. Let $\lambda>\kappa$ be a cardinal so that $\lambda=\beth_{\lambda}$, and let $\gamma=\beth_{\omega}(\lambda)$. Take $j: V \rightarrow M$ as an elementary embedding witnessing the $\gamma$ strongness of $\kappa$. Since $V \vDash " \delta$ is either $\alpha$ strong, $\alpha$ strongly compact, or $\alpha$ supercompact for every $\alpha<\kappa "$ and $\delta<\kappa, M \vDash " j(\delta)=\delta$ is either $\alpha$ strong, $\alpha$ strongly compact, or $\alpha$ supercompact for every $\alpha<j(\kappa)$ ". In particular, because $j(\kappa)>\gamma>\lambda, M \vDash " \delta$ is either $\lambda$ strong, $\lambda$ strongly compact, or $\lambda$ supercompact". As $V_{\gamma} \subseteq M, V \vDash$ " $\delta$ is either $\lambda$ strong, $\lambda$ strongly compact, or $\lambda$ supercompact" as well. Since $\lambda$ may be chosen arbitrarily large, this proves Lemma 1.1.

We observe that Lemma 1.1 has a local version. Specifically, if $\kappa$ is measurable and $\delta<\kappa$ is either $\alpha$ strong, $\alpha$ strongly compact, or $\alpha$ supercompact

2000 Mathematics Subject Classification: 03E35, 03E55.

Key words and phrases: supercompact cardinal, strongly compact cardinal, strong cardinal, non-reflecting stationary set of ordinals.

The author wishes to thank the referee for helpful suggestions that have been incorporated into this version of the paper. 
for $\alpha<\kappa$, then $\delta$ is either $\kappa+1$ strong, $\kappa$ strongly compact, or $\kappa$ supercompact. The proof is essentially the same as the one given above, with $j$ replaced by an elementary embedding witnessing $\kappa$ 's measurability, and the observation that the $\kappa$ closure of $M$ with respect to $V$ is enough to ensure that $\delta$ is either $\kappa+1$ strong, $\kappa$ strongly compact, or $\kappa$ supercompact in $V$.

Key to the proof of Lemma 1.1 is the fact that the inner model $M$ contains a large chunk of the universe $V$, something which will be true if $\kappa$ is either supercompact or, more weakly, strong. It is not necessarily the case, however, that if $\kappa$ is only strongly compact, then there is an elementary embedding witnessing any degree of strong compactness into an inner model $M$ containing any more of $V$ than $V_{\kappa+1}$. Thus, we can ask the following question: If $\kappa$ is a non-supercompact strongly compact cardinal and $\delta<\kappa$ is either $\alpha$ supercompact or $\alpha$ strong for every $\alpha<\kappa$, then must $\delta$ be either (fully) supercompact or strong? Note that by a theorem of Di Prisco [7], the answer to the analogue of this question if $\delta$ is $\alpha$ strongly compact for every $\alpha<\kappa$ is yes.

The purpose of this paper is to show that the answer to the above question is no. Specifically, we prove the following two theorems.

TheORem 1. Suppose $V \vDash " Z F C+\kappa_{1}<\kappa_{2}$ are supercompact". There is then a partial ordering $\mathbb{P} \in V$ so that $V^{\mathbb{P}} \vDash " Z F C+\kappa_{2}$ is strongly compact but not supercompact $+\kappa_{1}$ is $\alpha$ supercompact for every $\alpha<\kappa_{2}+$ $\kappa_{1}$ is not supercompact".

TheOREm 2. Suppose $V \vDash$ "ZFC $+\kappa$ is supercompact". There is then a partial ordering $\mathbb{P} \in V$ and a strong cardinal $\delta<\kappa$ so that $V^{\mathbb{P}} \vDash " Z F C+$ $\kappa$ is strongly compact but not supercompact $+\delta$ is $\alpha$ strong for every $\alpha<\kappa+$ $\delta$ is not strong".

Before giving the proofs of Theorems 1 and 2, we briefly mention some preliminary information. Essentially, our notation and terminology are standard, and when this is not the case, this will be clearly noted. For $\alpha<\beta$ ordinals, $[\alpha, \beta],[\alpha, \beta),(\alpha, \beta]$, and $(\alpha, \beta)$ are as in standard interval notation.

When forcing, $q \geq p$ will mean that $q$ is stronger than $p$. If $G$ is $V$-generic over $\mathbb{P}$, we will use both $V[G]$ and $V^{\mathbb{P}}$ to indicate the universe obtained by forcing with $\mathbb{P}$. If $x \in V[G]$, then $\dot{x}$ will be a term in $V$ for $x$. We may, from time to time, confuse terms with the sets they denote and write $x$ when we actually mean $\dot{x}$, especially when $x$ is some variant of the generic set $G$, or $x$ is in the ground model $V$.

If $\kappa$ is a cardinal and $\mathbb{P}$ is a partial ordering, $\mathbb{P}$ is $\kappa$-directed closed if for every cardinal $\delta<\kappa$ and every directed set $\left\langle p_{\alpha}: \alpha<\delta\right\rangle$ of elements of $\mathbb{P}$ (where $\left\langle p_{\alpha}: \alpha<\delta\right\rangle$ is directed if any two elements $p_{\varrho}$ and $p_{\nu}$ have a common upper bound of the form $p_{\sigma}$ ) there is an upper bound $p \in \mathbb{P}$. 
$\mathbb{P}$ is $\kappa$-strategically closed if in the two-person game in which the players construct an increasing sequence $\left\langle p_{\alpha}: \alpha \leq \kappa\right\rangle$, where player I plays odd stages and player II plays even and limit stages (choosing the trivial condition at stage 0 ), player II has a strategy which ensures the game can always be continued. Note that if $\mathbb{P}$ is $\kappa$-strategically closed and $f: \kappa \rightarrow V$ is a function in $V^{\mathbb{P}}$, then $f \in V$. $\mathbb{P}$ is $\prec \kappa$-strategically closed if in the two-person game in which the players construct an increasing sequence $\left\langle p_{\alpha}: \alpha<\kappa\right\rangle$, where player I plays odd stages and player II plays even and limit stages (again choosing the trivial condition at stage 0), player II has a strategy which ensures the game can always be continued.

Suppose $\kappa<\lambda$ are regular cardinals. A partial ordering $\mathbb{P}_{\kappa, \lambda}$ that will be used in this paper is the partial ordering for adding a non-reflecting stationary set of ordinals of cofinality $\kappa$ to $\lambda$. Specifically, $\mathbb{P}_{\kappa, \lambda}=\{s: s$ is a bounded subset of $\lambda$ consisting of ordinals of cofinality $\kappa$ so that for every $\alpha<\lambda, s \cap \alpha$ is non-stationary in $\alpha\}$, ordered by end-extension. Two things which can be shown (see [5] or [2]) are that $\mathbb{P}_{\kappa, \lambda}$ is $\delta$-strategically closed for every $\delta<\lambda$, and if $G$ is $V$-generic over $\mathbb{P}_{\kappa, \lambda}$, in $V[G]$, a non-reflecting stationary set $S=S[G]=\bigcup\left\{S_{p}: p \in G\right\} \subseteq \lambda$ of ordinals of cofinality $\kappa$ has been introduced. It is also virtually immediate that $\mathbb{P}_{\kappa, \lambda}$ is $\kappa$-directed closed.

We mention that we are assuming familiarity with the large cardinal notions of measurability, strongness, strong compactness, and supercompactness. Interested readers may consult [12] for further details. Also, unlike [12], we will say that the cardinal $\kappa$ is $\lambda$ strong for $\lambda>\kappa$ if there is $j: V \rightarrow M$ an elementary embedding having critical point $\kappa$ so that $j(\kappa)>\left|V_{\lambda}\right|$ and $V_{\lambda} \subseteq M$. As always, $\kappa$ is strong if $\kappa$ is $\lambda$ strong for every $\lambda>\kappa$.

2. The proof of Theorem 1. Let $V \vDash$ "ZFC $+\kappa_{1}<\kappa_{2}$ are supercompact". Without loss of generality, by first using an iteration of Laver's partial ordering of [13] (such as the one given in [1]) to force $\kappa_{i}$ for $i=1,2$ to have its supercompactness indestructible under $\kappa_{i}$-directed closed forcing, then employing an Easton support iteration to add to every measurable cardinal $\delta>\kappa_{2}$ a non-reflecting stationary set of ordinals of cofinality $\kappa_{2}$, and then forcing with a $\kappa_{1}$-directed closed partial ordering to ensure GCH holds at and above $\kappa_{1}$, we may also assume that $V \vDash$ "No cardinal $\lambda>\kappa_{2}$ is measurable $+\kappa_{1}$ 's supercompactness is indestructible under $\kappa_{1}$-directed closed forcing $+2^{\delta}=\delta^{+}$for every cardinal $\delta \geq \kappa_{1}$ ". The fact that no cardinal above $\kappa_{2}$ is measurable in $V$ follows from the Gap Forcing Theorem of [10] and [11].

Take now $\mathbb{P}_{0}$ as the Easton support iteration of length $\kappa_{2}$ which adds, to every measurable cardinal $\delta \in\left(\kappa_{1}, \kappa_{2}\right)$, a non-reflecting stationary set of ordinals of cofinality $\kappa_{1} . \mathbb{P}_{0}$ can be defined so as to have cardinality $\kappa_{2}$. Since $V \vDash$ "No cardinal $\lambda>\kappa_{2}$ is measurable $+2^{\delta}=\delta^{+}$for every cardinal 
$\delta \geq \kappa_{1}$ ", a theorem of Magidor (whose proof is given in Theorem 2 of [3]) tells us that $V^{\mathbb{P}_{0}} \vDash$ "There are no measurable cardinals in the interval $\left(\kappa_{1}, \kappa_{2}\right)$ $+\kappa_{2}$ is strongly compact". It then immediately follows that $V^{\mathbb{P}^{0}} \vDash$ " $\kappa_{2}$ is not $2^{\kappa_{2}}=\kappa_{2}^{+}$supercompact". Further, since $\mathbb{P}_{0}$, by its definition, is $\kappa_{1}$-directed closed, $V^{\mathbb{P}_{0}} \vDash$ " $\kappa_{1}$ is supercompact".

Work in $V_{0}=V^{\mathbb{P}^{0}}$. For the remainder of this paper, for $\alpha$ an arbitrary ordinal, let $\lambda_{\alpha}$ be the least measurable cardinal above $\alpha$. Since $V_{0} \vDash$ " $\kappa_{1}$ is supercompact $+\kappa_{2}$ is the least measurable cardinal above $\kappa_{1}$ ", by reflection, $A=\left\{\delta<\kappa_{1}: \delta\right.$ is $\lambda_{\delta}$ supercompact $\}$ is unbounded in $\kappa_{1}$. Therefore, we may define $\mathbb{P}_{1}$ in $V_{0}$ as the Easton support iteration of length $\kappa_{1}$ which first adds a Cohen subset of $\omega$ and then adds, to every $\delta \in A$, a non-reflecting stationary set of ordinals of cofinality $\omega$. In analogy to the definition of $\mathbb{P}_{0}, \mathbb{P}_{1}$ can be defined so as to have cardinality $\kappa_{1}$.

LEMMA 2.1. $V_{1}=V_{0}^{\mathbb{P}^{1}} \vDash " \kappa_{1}$ is $\alpha$ supercompact for every $\alpha<\kappa_{2}$ ".

Proof. Let $\eta<\kappa_{2}$ be an arbitrary inaccessible cardinal in the interval $\left(\kappa_{1}, \kappa_{2}\right)$, and let $j: V_{0} \rightarrow M$ be an elementary embedding witnessing the $\eta$ supercompactness of $\kappa_{1}$ so that $M \vDash$ " $\kappa_{1}$ is not $\eta$ supercompact". Since $\eta$ is below the least measurable cardinal above $\kappa_{1}, M \vDash " \kappa_{1}$ is not $\lambda_{\kappa_{1}}$ supercompact". This means $j\left(\mathbb{P}_{1}\right)=\mathbb{P}_{1} * \dot{\mathbb{Q}}$, where $\dot{\mathbb{Q}}$ is a term for a partial ordering that does not add a non-reflecting stationary set of ordinals of cofinality $\omega$ to $\kappa_{1}$, and the least $M$-cardinal above $\kappa_{1}$ to which $\dot{\mathbb{Q}}$ is forced to add a non-reflecting stationary set of ordinals of cofinality $\omega$ must also be above $\eta$.

Let $G_{0}$ be $V_{0}$-generic over $\mathbb{P}_{1}$, and let $H$ be $V_{0}\left[G_{0}\right]$-generic over $\mathbb{Q}$. Standard arguments show that $M\left[G_{0}\right]$ remains $\eta$ closed with respect to $V_{0}\left[G_{0}\right]$. Further, $j " G_{0} \subseteq G_{0} * H$. This means that in $V_{0}\left[G_{0}\right][H], j$ lifts to $j: V_{0}\left[G_{0}\right] \rightarrow M\left[G_{0}\right][H]$. By its definition, the closure properties of $M\left[G_{0}\right]$, and the last sentence of the preceding paragraph, $H$ is $V_{0}\left[G_{0}\right]$-generic over a partial ordering which is $\eta$-strategically closed in both $V_{0}\left[G_{0}\right]$ and $M\left[G_{0}\right]$. Therefore, $V_{0}\left[G_{0}\right] \vDash$ " $\kappa_{1}$ is $\alpha$ supercompact for every $\alpha<\eta$ ". Since $\eta$ was chosen as an arbitrary inaccessible cardinal in the interval $\left(\kappa_{1}, \kappa_{2}\right)$, this proves Lemma 2.1.

We remark that by the observation made immediately following the proof of Lemma 1.1, Lemma 2.1 actually shows that $\kappa_{1}$ is $\kappa_{2}$ supercompact in $V_{1}$.

LEMMA 2.2. $V_{1}=V_{0}^{\mathbb{P}^{1}} \vDash$ " $\kappa_{1}$ is not $2^{\kappa_{2}}=\kappa_{2}^{+}$supercompact".

Proof. We begin by noting that $V_{1}=V_{0}^{\mathbb{P}^{1}} \vDash " \kappa_{2}$ is the least measurable cardinal above $\kappa_{1}+\kappa_{2}$ is strongly compact but is not $2^{\kappa_{2}}=\kappa_{2}^{+}$supercompact". This follows by the fact $\kappa_{2}$ is both the least measurable and least strongly compact cardinal above $\kappa_{1}$ in $V_{0}$, the fact that $\mathbb{P}_{1}$ has cardinality $\kappa_{1}<\kappa_{2}$ in $V_{0}$, and the Lévy-Solovay results [14]. 
Work in $V_{0}$. For any $\alpha$, write $\mathbb{P}_{1}=\mathbb{Q}_{0} * \dot{\mathbb{Q}}_{1}$, where $\mathbb{Q}_{0}$ adds non-reflecting stationary sets of ordinals of cofinality $\omega$ to cardinals at most $\alpha$, and $\dot{\mathbb{Q}}_{1}$ is a term for the rest of $\mathbb{P}_{1}$. Since $\left|\mathbb{Q}_{0}\right| \leq 2^{\alpha}<\lambda_{\alpha}$, the results of [14] and the fact

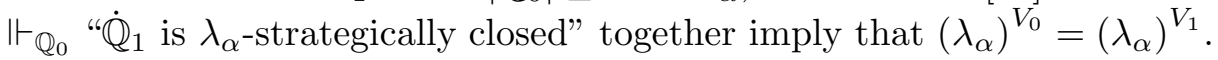

Write $\mathbb{P}_{1}=\mathbb{P}^{\prime} * \mathbb{P}^{\prime \prime}$, where $\left|\mathbb{P}^{\prime}\right|=\omega$ and $\vdash_{\mathbb{P}^{\prime}}$ " $\dot{\mathbb{P}}$ " is $\aleph_{1}$-strategically closed". In Hamkins' terminology of [9], [10], and [11], $\mathbb{P}_{1}$ "admits a gap at $\aleph_{1}$ ", so by the Gap Forcing Theorem of [10] and [11], any cardinal $\delta$ which is $\lambda_{\delta}$ supercompact in $V_{1}$ had to have been $\lambda_{\delta}$ supercompact in $V_{0}$. Since by its definition, forcing with $\mathbb{P}_{1}$ over $V_{0}$ destroys the weak compactness of any cardinal $\delta<\kappa_{1}$ that was $\lambda_{\delta}$ supercompact in $V_{0}$, the preceding sentence implies that $V_{1}=V_{0}^{\mathbb{P}^{1}} \vDash$ "No cardinal $\delta<\kappa_{1}$ is $\lambda_{\delta}$ supercompact". This immediately implies that $V_{1} \vDash " \kappa_{1}$ is not $2^{\kappa_{2}}=\kappa_{2}^{+}$supercompact", since otherwise, by choosing $k: V_{1} \rightarrow N$ as an elementary embedding witnessing the $2^{\kappa_{2}}$ supercompactness of $\kappa_{1}$ and reflecting the fact that $N \vDash " \kappa_{1}$ is $\kappa_{2}$ supercompact and $\kappa_{2}$ is the least measurable cardinal above $\kappa_{1}$ ", we would infer that $\left\{\delta<\kappa_{1}: \delta\right.$ is $\lambda_{\delta}$ supercompact $\}$ is unbounded in $\kappa_{1}$ in $V_{1}$. This proves Lemma 2.2.

By defining $\mathbb{P}=\mathbb{P}_{0} * \dot{\mathbb{P}}_{1}$, Lemmas 2.1 and 2.2 complete the proof of Theorem 1.

We conclude Section 2 with some observations. It is possible to change the definition of $\mathbb{P}_{1}$ so as to ensure $\kappa_{1}$ will satisfy a greater degree of supercompactness in $V_{1}$. If, e.g., we modify the definition of $\mathbb{P}_{1}$ so that we add non-reflecting stationary sets of ordinals of cofinality $\omega$ to every cardinal $\delta<\kappa_{1}$ which is $\beth_{\delta}\left(\lambda_{\delta}\right)$ supercompact (and by the supercompactness of $\kappa_{1}$, there are unboundedly in $\kappa_{1}$ many such cardinals), then in $V_{1}, \kappa_{1}$ will be $\beth_{\kappa_{1}}\left(\kappa_{2}\right)$ supercompact but not $2^{\left[\beth_{\kappa_{1}}\left(\kappa_{2}\right)\right]^{<\kappa_{1}}}=2^{\beth_{\kappa_{1}}\left(\kappa_{2}\right)}=\left(\beth_{\kappa_{1}}\left(\kappa_{2}\right)\right)^{+}$supercompact. However, due to the restrictions on the proof of Theorem 2 of [3], we need to know that $V \vDash$ "No cardinal $\lambda>\kappa_{2}$ is measurable". No such restrictions, however, are required in the proof of Theorem 2 of this paper, which we give below.

3. The proof of Theorem 2. Let $V \vDash$ "ZFC $+\kappa$ is supercompact". By Lemma 2.1 of [4] and the succeeding remark, we know that $\{\delta<\kappa: \delta$ is a strong cardinal $\}$ is unbounded in $\kappa$. Without loss of generality, by first forcing GCH, then choosing a strong cardinal $\delta<\kappa$, and then forcing with Gitik and Shelah's indestructibility partial ordering of [8] (which can be defined so as to have cardinality $\delta$ ), we may further assume that $V \vDash$ "GCH holds for cardinals at and above $\delta+\delta$ is a strong cardinal whose strongness is indestructible under forcing with an iteration of Prikry forcing as defined by Magidor in [15] which adds Prikry sequences to cardinals above $\delta$ ". 
Take now $\mathbb{P}_{0}$ as Magidor's iterated Prikry forcing of [15] which adds, to every measurable cardinal $\gamma \in(\delta, \kappa)$, a Prikry sequence. By the indestructibility properties of $V$ and Magidor's work of [15], $V^{\mathbb{P}_{0}}=V_{0} \vDash$ "GCH holds for cardinals at and above $\delta+\delta$ is a strong cardinal $+\kappa$ is strongly compact + There are no measurable cardinals in the interval $(\delta, \kappa)$ ". As in the proof of Theorem $1, V_{0} \vDash " \kappa$ is not $2^{\kappa}=\kappa^{+}$supercompact".

Work in $V_{0}$. Since $V_{0} \vDash$ " $\delta$ is strong $+\kappa$ is the least measurable cardinal above $\delta$ ", by reflection, $B=\left\{\gamma<\delta: \gamma\right.$ is $\lambda_{\gamma}$ strong $\}$ is unbounded in $\delta$. Therefore, in analogy to the proof of Theorem 1, we may define $\mathbb{P}_{1}$ in $V_{0}$ as the Easton support iteration which begins by adding a Cohen subset of $\omega$ and then adds, to every $\gamma \in B$, a non-reflecting stationary set of ordinals of cofinality $\omega$. As in the proof of Theorem $1, \mathbb{P}_{1}$ can be defined so as to have cardinality $\delta$. By the preceding paragraph, this has as an immediate consequence that in $V_{1}$, GCH holds for cardinals at and above $\delta$.

Lemma 3.1. $V_{0}^{\mathbb{P}_{1}}=V_{1} \vDash$ " $\delta$ is $\alpha$ strong for every $\alpha<\kappa$ ".

Proof. The proof is very similar to the proof of Lemma 2.5 of [4]. We use the notation and terminology from the introductory section of [6]. Fix $\eta>\delta, \eta<\kappa$ an inaccessible cardinal which is not also a Mahlo cardinal. Let $j: V_{0} \rightarrow M$ be an elementary embedding witnessing the $\eta+1$ strongness of $\delta$ generated by a $(\delta, \eta+1)$-extender of width $\delta$ so that $M \vDash$ " $\delta$ is not $\eta+1$ strong", and let $i: V_{0} \rightarrow N$ be the elementary embedding witnessing the measurability of $\delta$ generated by the normal ultrafilter $\mathcal{U}=\{x \subseteq \delta: \delta \in$ $j(x)\}$. We then have the commutative diagram

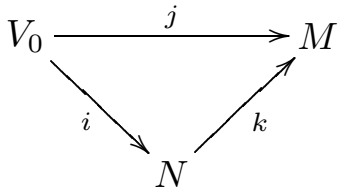

where $j=k \circ i$ and the critical point of $k$ is above $\delta$.

Since $\eta$ is below the least measurable cardinal above $\delta$ and $\eta$ is not a Mahlo cardinal, $M \vDash$ "There are no measurable cardinals in the interval $(\delta, \eta]+\delta$ is not $\lambda_{\delta}$ strong". Define $\varrho$ to be the least cardinal in $M$ above $\delta$ which is $\lambda_{\varrho}$ strong. By the next to last sentence, we can now infer that $\varrho>\eta$.

Define $f: \delta \rightarrow \delta$ as $f(\alpha)=$ The least inaccessible cardinal above $\lambda_{\alpha}$. By our choice of $\eta$ and the preceding paragraph, $\delta<\eta<j(f)(\delta)<\varrho$. Observe that $\varrho$ is also the least $M$-cardinal above $\delta$ to which $j\left(\mathbb{P}_{1}\right)$ adds a non-reflecting stationary set of ordinals of cofinality $\omega$.

Note now that $M=\left\{j(g)(a): a \in\left[\eta^{+}\right]^{<\omega}, \operatorname{dom}(g)=[\delta]^{|a|}, g:[\delta]^{|a|} \rightarrow V_{0}\right\}$ $=\left\{k(i(g))(a): a \in\left[\eta^{+}\right]^{<\omega}, \operatorname{dom}(g)=[\delta]^{|a|}, g:[\delta]^{|a|} \rightarrow V_{0}\right\}$. By defining $\gamma=$ $i(f)(\delta)$, we have $k(\gamma)=k(i(f)(\delta))=j(f)(\delta)>\eta^{+}$. This means $j(g)(a)=$ 
$k(i(g))(a)=k\left(i(g)\left\lceil[\gamma]^{|a|}\right)(a)\right.$, i.e., $M=\left\{k(h)(a): a \in\left[\eta^{+}\right]^{<\omega}, h \in N\right.$, $\left.\operatorname{dom}(h)=[\gamma]^{|a|}, h:[\gamma]^{|a|} \rightarrow N\right\}$. By elementariness, we must have $N \vDash$ " $\delta$ is not $\lambda_{\delta}$ strong and $\delta<\gamma=i(f)(\delta)<\delta_{0}=$ The least cardinal $\zeta$ in $N$ above $\delta$ which is $\lambda_{\zeta}$ strong $=$ The least cardinal to which $i\left(\mathbb{P}_{1}\right)-\delta$ adds a non-reflecting stationary set of ordinals of cofinality $\omega "$ ", since $M \vDash " k(\delta)=\delta$ is not $\lambda_{\delta}$ strong and $k(\delta)=\delta<k(\gamma)=k(i(f)(\delta))=j(f)(\delta)<k\left(\delta_{0}\right)=\varrho$ ". Therefore, $k$ can be assumed to be generated by an $N$-extender of width $\gamma \in\left(\delta, \delta_{0}\right)$.

Write $i\left(\mathbb{P}_{1}\right)=\mathbb{P}_{1} * \dot{\mathbb{Q}}_{0}$, where $\dot{\mathbb{Q}}_{0}$ is a term for the portion of $i\left(\mathbb{P}_{1}\right)$ adding non-reflecting stationary sets of ordinals of cofinality $\omega$ to $N$-cardinals in the interval $[\delta, i(\delta))$. Since $N \vDash " \delta$ is not $\lambda_{\delta}$ strong", $\dot{\mathbb{Q}}_{0}$ is actually a term for a partial ordering adding non-reflecting stationary sets of ordinals of cofinality $\omega$ to $N$-cardinals in the interval $(\delta, i(\delta))$, or more precisely, to $N$-cardinals in the interval $\left[\delta_{0}, i(\delta)\right)$.

Let $G_{0}$ be $V_{0}$-generic over $\mathbb{P}_{1}$. By the definition of $\mathbb{P}_{1}$ and the fact $\mathrm{GCH}$ holds in $V_{0}$ for cardinals at and above $\delta, N\left[G_{0}\right] \vDash "\left|\mathbb{Q}_{0}\right|=i(\delta)+\left|2^{\mathbb{Q}_{0}}\right|=$ $i\left(\delta^{+}\right)=(i(\delta))^{+}$". As $N$ is an ultrapower via a normal measure over $\delta$, this means $V_{0} \vDash "\left|(i(\delta))^{+}\right|=\delta^{+}$", so we can let $\left\langle D_{\alpha}: \alpha<\delta^{+}\right\rangle \in V_{0}\left[G_{0}\right]$ be an enumeration of the dense open subsets of $\mathbb{Q}_{0}$ present in $N\left[G_{0}\right]$. For the purposes of the argument to be given below, we also assume that $\left\langle D_{\alpha}\right.$ : $\left.\alpha<\delta^{+}\right\rangle$has been defined so that for every dense open subset $D \subseteq \mathbb{Q}_{0}$ found in $N\left[G_{0}\right]$, for some odd ordinal $\beta+1, D=D_{\beta+1}$. Further, since $V_{0} \vDash "\left|\mathbb{P}_{1}\right|=\delta$ ", standard arguments show that $N\left[G_{0}\right]$ remains $\delta$ closed with

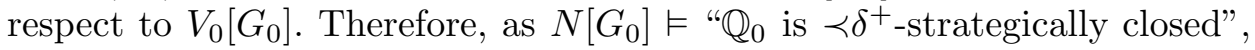
this fact is true in $V_{0}\left[G_{0}\right]$ as well.

We can now construct an $N\left[G_{0}\right]$-generic object, $G_{1}^{*}$, in $V_{0}\left[G_{0}\right]$ as follows. Players I and II play a game of length $\delta^{+}$. The initial pair of moves is generated by player II choosing the trivial condition $q_{0}$ and player I responding by choosing $q_{1} \in D_{1}$. Then, at an even stage $\alpha+2$, player II picks $q_{\alpha+2} \geq q_{\alpha+1}$ by using some fixed strategy $\mathcal{S}$, where $q_{\alpha+1}$ was chosen by player I to be so that $q_{\alpha+1} \in D_{\alpha+1}$ and $q_{\alpha+1} \geq q_{\alpha}$. If $\alpha$ is a limit ordinal, player II uses $\mathcal{S}$ to pick $q_{\alpha}$ extending each $q_{\beta}$ for $\beta<\alpha$. By the $\prec \delta^{+}$-strategic closure of $\mathbb{Q}_{0}$ in both $N\left[G_{0}\right]$ and $V\left[G_{0}\right]$, the sequence $\left\langle q_{\alpha}: \alpha<\delta^{+}\right\rangle$as just described exists. By construction, $G_{1}^{*}=\left\{p \in \mathbb{Q}_{0}: \exists \alpha<\delta^{+}\left[q_{\alpha} \geq p\right]\right\}$ is our $N\left[G_{0}\right]$-generic object over $\mathbb{Q}_{0}$. Since $i^{\prime \prime} G_{0} \subseteq G_{0} * G_{1}^{*}, i$ lifts to $i: V_{0}\left[G_{0}\right] \rightarrow N\left[G_{0}\right]\left[G_{1}^{*}\right]$, and since $k^{\prime \prime} G_{0}=G_{0}$ and $k(\delta)=\delta, k$ lifts to $k: N\left[G_{0}\right] \rightarrow M\left[G_{0}\right]$. By Fact 3 of Section 1.2 .2 of $[6], k: N\left[G_{0}\right] \rightarrow M\left[G_{0}\right]$ can also be assumed to be generated by an extender of width $\gamma \in\left(\delta, \delta_{0}\right)$.

In analogy to the above, write $j\left(\mathbb{P}_{1}\right)=\mathbb{P}_{1} * \dot{\mathbb{Q}}_{1}$. By the last sentence of the preceding paragraph and the fact $\delta_{0}$ is the least $N$-cardinal to which $\dot{\mathbb{Q}}_{0}$ is forced to add a non-reflecting stationary set of ordinals of cofinality $\omega$, we can use Fact 2 of Section 1.2.2 of [6] to infer that $H=\left\{p \in \mathbb{Q}_{1}\right.$ : 
$\left.\exists q \in k^{\prime \prime} G_{1}^{*}[q \geq p]\right\}$ is $M\left[G_{0}\right]$-generic over $k\left(\mathbb{Q}_{0}\right)=\mathbb{Q}_{1}$. Thus, $k$ lifts to $k: N\left[G_{0}\right]\left[G_{1}^{*}\right] \rightarrow M\left[G_{0}\right][H]$, and we get the new commutative diagram

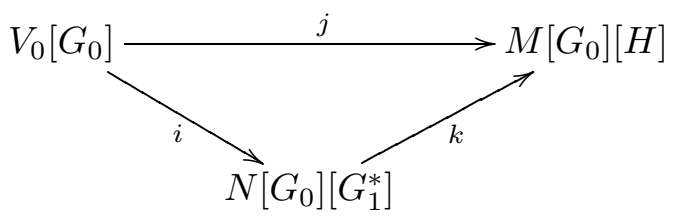

Since $\varrho>\eta$, the $M$-cardinals to which $\dot{\mathbb{Q}}_{1}$ is forced to add non-reflecting stationary sets of ordinals of cofinality $\omega$ lie in the interval $\left(\eta^{+}, j(\delta)\right)$. Therefore, as $V_{\eta+1} \subseteq M, V_{\eta+1}\left[G_{0}\right] \subseteq M\left[G_{0}\right]$, and as $\mathbb{Q}_{1}$ adds non-reflecting stationary sets of ordinals of cofinality $\omega$ to certain inaccessible $M$-cardinals in the interval $\left(\eta^{+}, j(\delta)\right), V_{\eta+1}\left[G_{0}\right]$ is the set of all sets of rank below $\eta+1$ in $M\left[G_{0}\right][H]$. Hence, $j$ is an $\eta+1$ strong embedding. Since $\eta$ was an arbitrary non-Mahlo inaccessible cardinal below $\kappa$, this proves Lemma 3.1.

We remark that by the observation made immediately following the proof of Lemma 1.1, Lemma 3.1 actually shows that $\delta$ is $\kappa+1$ strong in $V_{1}$.

\section{LEMMA 3.2. $V_{1} \vDash " \delta$ is not $\kappa+2$ strong".}

Proof. We argue in analogy to the proof of Lemma 2.2. We again begin by noting that $V_{1}=V_{0}^{\mathbb{P}^{1}} \vDash " \kappa$ is the least measurable cardinal above $\delta+$ $\kappa$ is strongly compact but is not $2^{\kappa}=\kappa^{+}$supercompact". This follows by the fact $\kappa$ is both the least measurable and least strongly compact cardinal above $\delta$ in $V_{0}$, the fact that $\mathbb{P}_{1}$ has cardinality $\delta<\kappa$ in $V_{0}$, and the results of [14].

Work in $V_{0}$. As in the proof of Lemma 2.2, for any ordinal $\alpha,\left(\lambda_{\alpha}\right)^{V_{0}}=$ $\left(\lambda_{\alpha}\right)^{V_{1}}$. Also, we can once more write $\mathbb{P}_{1}=\mathbb{P}^{\prime} * \dot{\mathbb{P}}^{\prime \prime}$, where $\left|\mathbb{P}^{\prime}\right|=\omega$ and $\Vdash_{\mathbb{P}^{\prime}}$ " $\dot{\mathbb{P}}$ " is $\aleph_{1}$-strategically closed". As before, $\mathbb{P}_{1}$ "admits a gap at $\aleph_{1}$ ", so by the Gap Forcing Theorem of [10] and [11], any cardinal $\zeta$ which is $\lambda_{\zeta}$ strong in $V_{1}$ had to have been $\lambda_{\zeta}$ strong in $V_{0}$. Since by its definition, forcing with $\mathbb{P}_{1}$ over $V_{0}$ destroys the weak compactness of any cardinal $\zeta<\delta$ that was $\lambda_{\zeta}$ strong in $V_{0}$, the preceding sentence implies that $V_{1}=V_{0}^{\mathbb{P}^{1}} \vDash$ "No cardinal $\zeta<\delta$ is $\lambda_{\zeta}$ strong". This immediately implies that $V_{1} \vDash$ " $\delta$ is not $\kappa+2$ strong", since otherwise, by choosing $\ell: V_{1} \rightarrow M^{*}$ as an elementary embedding witnessing the $\kappa+2$ strongness of $\delta$ and reflecting the fact that $M^{*} \vDash " \delta$ is $\kappa$ strong and $\kappa$ is the least measurable cardinal above $\delta$ ", we would infer that $\left\{\zeta<\delta: \zeta\right.$ is $\lambda_{\zeta}$ strong $\}$ is unbounded in $\delta$ in $V_{1}$. This proves Lemma 3.2.

By defining $\mathbb{P}=\mathbb{P}_{0} * \dot{\mathbb{P}}_{1}$, Lemmas 3.1 and 3.2 complete the proof of Theorem 2. 
We conclude Section 3 and this paper with several observations. First, as the referee has essentially indicated, if $V \vDash$ "ZFC $+\mathrm{GCH}+\delta<\kappa$ are so that $\delta$ is strong and $\kappa$ is strongly compact", then we may force over $V$ with the partial ordering $\mathbb{P}$ as just defined in order to obtain the conclusions of Theorem 2. In addition, as before, it is possible to change the definition of $\mathbb{P}_{1}$ so as to ensure $\delta$ will satisfy a greater degree of strongness in $V_{1}$. If, e.g., we change the definition of $\mathbb{P}_{1}$ so that we add non-reflecting stationary sets of ordinals of cofinality $\omega$ to every cardinal $\zeta<\delta$ which is $\beth_{\zeta}\left(\lambda_{\zeta}\right)$ strong (and by the strongness of $\delta$, there are unboundedly in $\delta$ many such cardinals), then in $V_{1}, \delta$ will be $\beth_{\delta}(\kappa)$ strong but not $\beth_{\delta}(\kappa)+1$ strong. Also, since Magidor's proof from [15] that iterated Prikry forcing preserves the strong compactness of $\kappa$ is valid regardless of the large cardinal structure of the universe above $\kappa$, unlike Theorem 1, there is no need to do an initial forcing to ensure that $V \vDash$ "No cardinal $\lambda>\kappa$ is measurable".

Finally, we note that under the same hypotheses as in Theorem 1, i.e., that $V \vDash$ "ZFC $+\kappa_{1}<\kappa_{2}$ are supercompact", it is possible to modify the definition of the partial ordering $\mathbb{P}$ of Theorem 1 so that $V^{\mathbb{P}} \vDash$ "ZFC $+\kappa_{2}$ is strongly compact but not supercompact $+\kappa_{1}$ is $\alpha$ supercompact for every $\alpha<\kappa_{2}+\kappa_{1}$ is not supercompact $+\kappa_{1}$ is strong". To do this, we observe that Lemma 2.1 of [4] and the succeeding remark actually imply that if $j: V \rightarrow M$ is an elementary embedding witnessing (at least) the $2^{\lambda_{\kappa_{1}}}$ supercompactness of $\kappa_{1}$, then $M \vDash " \kappa_{1}$ is a strong cardinal and $\kappa_{1}$ is $\lambda_{\kappa_{1}}$ supercompact", meaning that $A=\left\{\delta<\kappa_{1}: \delta\right.$ is a strong cardinal and $\delta$ is $\lambda_{\delta}$ supercompact $\}$ is unbounded in $\kappa_{1}$. Therefore, if $\mathbb{P}_{0}$ is as in the definition given in the proof of Theorem $1, V_{0}=V^{\mathbb{P}_{0}}$, and $\mathbb{P}_{1}$ is defined in $V_{0}$ as the Easton support iteration of length $\kappa_{1}$ which first adds a Cohen subset of $\omega$ and then adds, to every $\delta \in A$, a non-reflecting stationary set of ordinals of cofinality $\omega$, the exact same arguments as before show that $V^{\mathbb{P}} \vDash$ "ZFC + $\kappa_{2}$ is strongly compact but not supercompact $+\kappa_{1}$ is $\alpha$ supercompact for every $\alpha<\kappa_{2}$ ". If in the proof of Lemma 2.2, we replace the property " $\delta$ is $\lambda_{\delta}$ supercompact" with " $\delta$ is a strong cardinal and $\delta$ is $\lambda_{\delta}$ supercompact", then the same proof as given in Lemma 2.2 remains valid and shows $V^{\mathbb{P}} \vDash$ " $\kappa_{1}$ is not $2^{\kappa_{2}}=\kappa_{2}^{+}$supercompact". Further, if we choose $\lambda>\kappa_{2}$ as any cardinal so that $\lambda=\aleph_{\lambda}=\beth_{\lambda}$ and $j: V_{0} \rightarrow M$ as an elementary embedding witnessing the $\lambda$ strongness of $\kappa_{1}$ so that $M \vDash$ " $\kappa_{1}$ is not $\lambda$ strong", then either the argument given in the proof of Lemma 2.5 of [4] or the one in the proof of Lemma 3.1 shows that $V_{0}^{\mathbb{P}_{1}}=V^{\mathbb{P}} \vDash " \kappa_{1}$ is $\lambda$ strong". Since $\lambda$ may be chosen arbitrarily large, this means that $V^{\mathbb{P}} \vDash " \kappa_{1}$ is strong". And, in analogy to what was mentioned in the concluding remarks of Section 2, it is possible to change the definition of $\mathbb{P}_{1}$ to ensure that $\kappa_{1}$ witnesses a greater degree of supercompactness in $V^{\mathbb{P}}$, assuming that the cardinals to which non-reflecting stationary sets of ordinals of cofinality $\omega$ are added are also strong. 


\section{References}

[1] A. Apter, Laver indestructibility and the class of compact cardinals, J. Symbolic Logic 63 (1998), 149-157.

[2] - Strong compactness, measurability, and the class of supercompact cardinals, Fund. Math. 167 (2001), 65-78.

[3] A. Apter and J. Cummings, Identity crises and strong compactness, J. Symbolic Logic 65 (2000), 1895-1910.

[4] -, 一, Identity crises and strong compactness II: strong cardinals, Arch. Math. Logic 40 (2001), 25-38.

[5] J. Burgess, Forcing, in: J. Barwise (ed.), Handbook of Mathematical Logic, NorthHolland, Amsterdam, 1977, 403-452.

[6] J. Cummings, A model in which GCH holds at successors but fails at limits, Trans. Amer. Math. Soc. 329 (1992), 1-39.

[7] C. Di Prisco and J. Henle, On the compactness of $\aleph_{1}$ and $\aleph_{2}$, J. Symbolic Logic 43 (1978), 394-401.

[8] M. Gitik and S. Shelah, On certain indestructibility of strong cardinals and a question of Hajnal, Arch. Math. Logic 28 (1989), 35-42.

[9] J. D. Hamkins, Destruction or preservation as you like it, Ann. Pure Appl. Logic 91 (1998), 191-229.

[10] —, Gap forcing, Israel J. Math. 125 (2001), 237-252.

[11] —, Gap forcing: generalizing the Lévy-Solovay theorem, Bull. Symbolic Logic 5 (1999), 264-272.

[12] A. Kanamori, The Higher Infinite, Springer, Berlin, 1994.

[13] R. Laver, Making the supercompactness of $\kappa$ indestructible under $\kappa$-directed closed forcing, Israel J. Math. 29 (1978), 385-388.

[14] A. Lévy and R. Solovay, Measurable cardinals and the continuum hypothesis, ibid. 5 (1967), 234-248.

[15] M. Magidor, How large is the first strongly compact cardinal?, Ann. Math. Logic 10 (1976), 33-57.

[16] - On the role of supercompact and extendible cardinals in logic, Israel J. Math. 10 (1971), 147-157.

Department of Mathematics

Baruch College of CUNY

New York, NY 10010, U.S.A.

E-mail: awabb@cunyvm.cuny.edu

http://math.baruch.cuny.edu/ ${ }^{\sim}$ apter

Received 24 November 2001;

in revised form 24 February 2002 